\title{
Preventive HIPEC in combination with perioperative FLOT versus FLOT alone for resectable diffuse type gastric and gastroesophageal junction type II/III adenocarcinoma - the phase III "PREVENT"- (FLOT9) trial of the AIO /CAOGI /ACO
}

Thorsten O. Götze ${ }^{1,2}$, Pompiliu Piso ${ }^{3}$, Sylvie Lorenzen ${ }^{4}$, Ulli S. Bankstahl ${ }^{1 *}$ (D, Claudia Pauligk², Moustafa Elshafei ${ }^{5}$, Giuseppe Amato ${ }^{6}$, Daniel Reim ${ }^{7}$, Wolf O. Bechstein ${ }^{8}$, Alfred Königsrainer ${ }^{9}$, Stefan P. Mönig ${ }^{10}$, Beate Rau ${ }^{11}$, Matthias Schwarzbach ${ }^{12}$ and Salah-Eddin Al-Batran ${ }^{1,2}$

\begin{abstract}
Background: The main reason for treatment failure after curative surgical resection of gastric cancer is intraabdominal spread, with $40-50 \%$ peritoneal seeding as primary localization of recurrence. Peritoneal relapse is seen in $60-70 \%$ of tumors of diffuse type, compared to only $20-30 \%$ of intestinal type.

Hyperthermic IntraPEritoneal Chemoperfusion (HIPEC) is an increasingly used therapy method for patients with peritoneal metastases. The preventive use of HIPEC could represent an elegant approach for patients (pts) before macroscopic peritoneal seeding, since pts. with operable disease are fit and may have potential risk of microscopic involvement, thus having a theoretical chance of cure with HIPEC even without the need for cytoreduction. No results from a PCRT from the Western hemisphere have yet been published.
\end{abstract}

Methods: This is a multicenter, randomized, controlled, open-label study including a total of 200 pts. with localized and locally advanced diffuse or mixed type (Laurens's classification) adenocarcinoma of the stomach and Type II/III GEJ.

All enrolled pts. will have received 3-6 pre-operative cycles of biweekly FLOT (Docetaxel $50 \mathrm{mg} / \mathrm{m}^{2}$; Oxaliplatin $85 \mathrm{mg} / \mathrm{m}^{2}$; Leucovorin $200 \mathrm{mg} / \mathrm{m}^{2} ; 5-\mathrm{FU} 2600 \mathrm{mg} / \mathrm{m}^{2}$, q2wk).

Pts will be randomized 1:1 to receive surgery only and postoperative FLOT (control arm) or surgery + intraoperative HIPEC (cisplatin $75 \mathrm{mg} / \mathrm{m}^{2}$ solution administered at a temperature of $42^{\circ} \mathrm{C}$ for $90 \mathrm{~min}$ ) and postoperative FLOT (experimental arm). Surgery is carried out as gastrectomy or transhiatal extended gastrectomy. Primary endpoint is PFS/DFS, major secondary endpoints are OS, rate of pts. with peritoneal

\footnotetext{
* Correspondence: bankstahl.ulli@khnw.de

'Institute of Clinical Cancer Research (IKF) at Krankenhaus Nordwest,

UCT-University Cancer Center, Frankfurt, Germany

Full list of author information is available at the end of the article
}

(c) The Author(s). 2021 Open Access This article is licensed under a Creative Commons Attribution 4.0 International License, which permits use, sharing, adaptation, distribution and reproduction in any medium or format, as long as you give appropriate credit to the original author(s) and the source, provide a link to the Creative Commons licence, and indicate if changes were made. The images or other third party material in this article are included in the article's Creative Commons licence, unless indicated otherwise in a credit line to the material. If material is not included in the article's Creative Commons licence and your intended use is not permitted by statutory regulation or exceeds the permitted use, you will need to obtain permission directly from the copyright holder. To view a copy of this licence, visit http://creativecommons.org/licenses/by/4.0/ The Creative Commons Public Domain Dedication waiver (http://creativecommons.org/publicdomain/zero/1.0/) applies to the data made available in this article, unless otherwise stated in a credit line to the data. 
relapse at 2 and 3 years, perioperative morbidity/mortality and quality of life.

The trial starts with a safety run-in phase. After 20 pts. had curatively intended resection in Arm B, an interim safety analysis is performed.

Recruitment has already started and first patient in was on January 18th, 2021.

Discussion: If the PREVENT concept proves to be effective, this could potentially lead to a new standard of therapy. On the contrary, if the outcome is negative, pts. with gastric cancer and no peritoneal involvement will not be treated with HIPEC during surgery.

Trial registration: The study is registered on June 25th, 2020 under ClinicalTrials.gov Identifier: NCT04447352; EudraCT: 2017-003832-35.

Keywords: Gastric cancer, Gastroesophageal junction cancer, Lauren- classification, Signet ring cells, HIPEC, Hyperthermic intraperitoneal chemotherapy, FLOT- regimen, Gastrectomy, Quality of life, Cisplatin, Peritoneal carcinomatosis

\section{Background}

The main reason for treatment failure after curative surgical resection of gastric cancer is intra-abdominal spread. Local recurrence, retroperitoneal lymph node metastases, peritoneal seeding and liver metastases are observed in about $90 \%$ of patients having tumor progression. Most recurrences are reported within 18 months from the primary surgical resection. In $40-50 \%$ of these cases, a peritoneal seeding is the primary localization of recurrence. The likelihood for a peritoneal relapse is even much more common in the diffuse type gastric cancer according to Lauren's classification, and ranges between 60 and 70\% [1-5]. On the other hand, intestinal type tumors tend to spread via hematogenous routes and show only a peritoneal seeding rate of $20-30 \%$.

Therefore, the outcome of diffuse type respectively signet-ring cell gastric cancer including the mixed types acc. to Lauren, remains unsatisfactory. Signet ring cell gastric cancer is associated with younger age; usually affects the corpus of the stomach and presents rapid relapse and worse prognosis compared with the intestinal type. Moreover, the response of peritoneal metastases to systemic chemotherapy is poor, mainly due to the presence of a so called "plasma-peritoneal barrier" which isolates the peritoneal cavity from the effects of intravenous chemotherapy [6].

Taken together, considerable investigation is still required to improve perioperative protocols, particularly the intra-operative component, in this aggressive subgroup of gastric cancer.

FLOT, a docetaxel-based triplet combination consisting of 5-FU, leucovorin, oxaliplatin and docetaxel, (Docetaxel $50 \mathrm{mg} / \mathrm{m}^{2}$ in $250 \mathrm{ml} \mathrm{NaCl} 0.9 \%$, iv over $1 \mathrm{~h}$; Oxaliplatin $85 \mathrm{mg} / \mathrm{m}^{2}$ in $500 \mathrm{ml} \mathrm{G5 \%}$, iv over $2 \mathrm{~h}$; Leucovorin $200 \mathrm{mg} / \mathrm{m}^{2}$ in $250 \mathrm{ml} \mathrm{NaCl} 0.9 \%$, iv over $30 \mathrm{~min}$; 5 -FU $2600 \mathrm{mg} / \mathrm{m}^{2}$, iv over $24 \mathrm{~h}, \mathrm{q} 2 \mathrm{wk}$ ) [7, 8] is one of the most intensively evaluated regimens for gastric and GEJ adenocarcinoma. It has been evaluated in the metastatic setting [9], in the limited metastatic setting [10], in elderly patients [11] and in operable patients [7]. The AIO FLOT4 phase II/III study has evaluated FLOT versus Epirubicin, Cisplatin and 5-FU (ECF) as well Epirubicin, Cisplatin and Xeloda (ECX) $(n=716)$. The phase II part of the randomized phase II/III FLOT4 trial regarding histopathological regression [7] comprised 300 patients, of whom 265 patients were evaluable on an intent-to-treat basis. FLOT was associated with significantly higher proportions of patients achieving pathological complete regression than ECF/ECX (20 [16\%; $95 \%$ CI $10-23$ ] of 128 patients vs 8 [6\%;3-11] of 137 patients; $p=0.02$ ). Also the rate of complete or subtotal regression (TRG1a/b) was significantly higher with FLOT (47 [37\%] of 128 vs. 31 [23\%] of 137, $\mathrm{p}=0.02$ ). The differences were more pronounced in intestinal type tumors. Despite the problems of systemic chemotherapy in diffuse type gastric cancers, FLOT was able the show efficacy in this type of gastric cancers according to the phase III data of the FLOT4 trial [8]. FLOT is regarded a standard chemotherapy regimen for gastric cancer in the perioperative setting. So FLOT is also regarded as the most effective backbone protocol for the current multimodal trial for patients suffering from diffuse type (acc. to Lauren) gastric or GEJ adenocarcinoma.

Hyperthermic Intraperitoneal Chemoperfusion (HIPEC) is an increasingly used therapy method for patients with peritoneal metastases. Although some evidence exists on its efficacy for selected disease entities it could not become standard of care due to lack of randomized trials. Moreover, patients with macroscopic involvement of the peritoneal cavity are less likely to be cured, leading many physicians to avoid burdensome therapy approaches including HIPEC for these patients. In contrast, the preventive use of HIPEC could represent a more elegant approach, since patients with operable disease are fit and have no macroscopic visible peritoneal involvement, thus having a theoretical chance of cure. This would justify 
more intense therapy regimen. Unfortunately, current data regarding HIPEC- procedure in different tumor entities are contradictory.

Among patients with a stage III epithelial ovarian cancer, the addition of cisplatin based HIPEC to cytoreductive surgery resulted in an improvement of recurrencefree and overall survival compared to surgery alone and established the role of HIPEC in ovarian cancer entity as more or less a standard procedure in stage III patients based on phase III data [12].

On the other hand, in colorectal cancer data regarding intraperitoneal therapy with HIPEC are currently not as promising as in the ovarian entity. PROPHYLOCHIPPRODIGE 15 showed that systematic second-look surgery including oxaliplatin based HIPEC-therapy did not improve disease-free survival compared with standard surveillance only [13]. In addition, PRODIGE- 7 showed also no benefit in overall survival after adding HIPEC to cytoreductive surgery with this combination in colorectal cancer with an existing peritoneal seeding and a Peritoneal Cancer Index of 25 or less and the intent for a curative approach in a metastatic disease [14].

A registry study considering 152 patients [15] could show that in cytoreductive surgeries for peritoneal metastases in small bowel adenocarcinoma combined with the HIPEC procedure achieved prolonged survival for selected patients with acceptable morbidity and mortality.

Nevertheless, HIPEC- procedure is still an established procedure according e.g. to the S3- guidelines in Germany [16] in metastatic colorectal cancer patients, but based on the French data a matter of debate [17].

Glehen et al. published a report on the French experience in the curative treatment of gastric peritoneal carcinomatosis [18], in a multi-institutional study of 159 patients treated by cytoreductive surgery combined with perioperative intraperitoneal chemotherapy. The therapeutic approach combining cytoreductive surgery with intraperitoneal chemotherapy for patients with gastric carcinomatosis achieved a long-term survival in a selected group of patients with only limited and resectable peritoneal carcinomatosis. In addition, the trial showed an increased mortality rate. The high mortality rate underlines necessarily strict selection that should be reserved to experienced institutions involved in the management of peritoneal carcinomatosis and gastric surgery. The current PREVENT (FLOT9) - trial is only performed at selected high- volume HIPEC- centers with an expertise in the field.

Based on the negative data for HIPEC- protocols based on intraperitoneal oxaliplatin $[13,14]$ and the positive data for cisplatin [12], there is a strong rationale for the use of cisplatin instead of oxaliplatin in new HIPECprotocols, e.g. in our protocol of PREVENT (FLOT9).
We believe that there is a strong theoretical rationale for the conduct of a randomized study evaluating the efficacy and safety of preventive intraperitoneal cisplatin based HIPEC in combination with systemic upfront FLOT - regimen (currently the most effective chemotherapy backbone protocol) in the perioperative treatment of patients with resectable adenocarcinoma with a diffuse or mixed type according to Lauren's- classification of the stomach or GEJ Type II/III without signs of a systemic, especially a peritoneal seeding.

\section{Methods/design \\ Protocol overview}

The PREVENT (FLOT9) study is a multicenter, randomized, controlled and open-label study including patients with localized and locally advanced adenocarcinoma of the stomach and type II/III GEJ of diffuse or mixed type according to Lauren's classification, scheduled to receive perioperative chemotherapy combined with or without intraoperative HIPEC procedure.

The scope of the trial is to evaluate the efficacy as well as the safety and tolerability of the combination of perioperative chemotherapy with an intraoperative HIPEC for resectable diffuse or mixed type gastric and GEJ (types II/III) adenocarcinoma.

Patients with localized and locally advanced diffuse or mixed type adenocarcinoma of the stomach and type II/ III GEJ (i.e. $\geq \mathrm{cT} 3$ any $\mathrm{N}$ or any $\mathrm{T} \mathrm{N}$-positive) with laparoscopic exclusion of peritoneal seeding and radiological exclusion of other distant metastases will be included in this trial after a central review by medical and surgical oncologist.

All enrolled patients will have received 3-6 preoperative cycles (de-escalation or dose modification allowed) of biweekly FLOT (Docetaxel $50 \mathrm{mg} / \mathrm{m}^{2}$ in 250 $\mathrm{ml} \mathrm{NaCl} 0.9 \%$, iv over $1 \mathrm{~h}$; Oxaliplatin $85 \mathrm{mg} / \mathrm{m}^{2}$ in 500 $\mathrm{ml} \mathrm{G5 \%}$, iv over $2 \mathrm{~h}$; Leucovorin $200 \mathrm{mg} / \mathrm{m}^{2}$ in $250 \mathrm{ml}$ $\mathrm{NaCl} 0.9 \%$, iv over $30 \mathrm{~min}$; 5 -FU $2600 \mathrm{mg} / \mathrm{m}^{2}$, iv over 24 $\mathrm{h}, \mathrm{q} 2 \mathrm{wk}$ ) in the preoperative treatment phase. After completion of neoadjuvant FLOT- therapy followed by pre-operative tumor assessment, patients without disease progression (expected to be approximately $90 \%$ of the patients) will be included into the trial, stratified by study site, histology type of tumor (Lauren classification diffuse vs. mixed) and initial clinical stage $(\mathrm{N}-\mathrm{vs} . \mathrm{N}+)$. Pts. will be randomized 1:1 to receive either standard of care (SOC) surgery plus postoperative FLOT- regimen (Arm A- standard) or SOC- surgery combined with intraoperative cisplatin based HIPEC- procedure followed by postoperative FLOT- therapy (Arm B- experimental). Randomization will be performed electronically in the eCRF by the site staff using variance minimization, so the sequence of randomization results is not known to the investigators. 


\section{Arm A (FLOT- standard arm)}

Surgery in Arm A is planned to occur 4 to 6 weeks after d1 of last FLOT cycle. Surgery is carried out in kind of standardized gastrectomy or transhiatal extended gastrectomy, both including D2- lymphadenectomy. Patients will receive 4 additional post-operative cycles $(8$ weeks) of FLOT (Docetaxel $50 \mathrm{mg} / \mathrm{m}^{2}$ in $250 \mathrm{ml} \mathrm{NaCl}$ $0.9 \%$, iv over $1 \mathrm{~h}$; Oxaliplatin $85 \mathrm{mg} / \mathrm{m}^{2}$ in $500 \mathrm{ml} \mathrm{G} 5 \%$, iv over $2 \mathrm{~h}$; Leucovorin $200 \mathrm{mg} / \mathrm{m}^{2}$ in $250 \mathrm{ml} \mathrm{NaCl} 0.9 \%$, iv over $30 \mathrm{~min}$; 5 -FU $2600 \mathrm{mg} / \mathrm{m}^{2}$, iv over $24 \mathrm{~h}$, q2wk) in the post-operative treatment phase. Post-operative treatment should start 6 to 8 weeks, but at maximum 12 weeks, after surgery.

\section{Arm B (FLOT/ HIPEC- experimental arm)}

Surgery in Arm B is planned to occur 4 to 6 weeks after d1 of last FLOT- cycle. Surgery is carried out in kind of gastrectomy or transhiatal extended gastrectomy, both including D2- lymphadenectomy. Surgery will be combined with an intraoperative Hyperthermic Intraperitoneal Chemoperfusion (HIPEC).

HIPEC itself can be performed in closed or openabdomen procedure, according to the local standards at study site. The protocols advices the prevention of nephrotoxicity during hyperthermic perfusion with cisplatin. Before hyperthermic perfusion starts, urine production should be more or equal than $1 \mathrm{ml} / \mathrm{kg} / \mathrm{hr}$. We recommend the usage of sodium thiosulfate, but the usage depends on the local preferences and standards. At start of hyperthermic perfusion: Sodium thiosulfate: $9 \mathrm{~g} / \mathrm{m} 2$ in $200 \mathrm{ml}$ distilled water, made isotone with sodium chloride, is to be given IV push over $15-20 \mathrm{~min}$, concurrently at start of hyperthermic infusion of cisplatin. This is to be followed by $12 \mathrm{~g} / \mathrm{m} 2$ thiosulfate IV continuous infusion over $6 \mathrm{~h}$ (the $12 \mathrm{~g} / \mathrm{m} 2$ should be dissolved in 11 of distilled water and infused at $167 \mathrm{ml} / \mathrm{hr}$ ). After positioning of inflow catheter and drains intraabdominal cisplatin solution $(75 \mathrm{mg} / \mathrm{m} 2$ in $\mathrm{NaCl} 0.9 \%)$ will be administered at a temperature of $42^{\circ} \mathrm{C}$ for $90 \mathrm{~min}$. Perfusion with cisplatin at a dose of $75 \mathrm{mg}$ per square meter and at a flow rate of 11 per minute will then be initiated (with $50 \%$ of the dose perfused initially, $25 \%$ at $30 \mathrm{~min}$, and $25 \%$ at $60 \mathrm{~min}$ ). The perfusion volume will be adjusted such that the entire abdomen is exposed to the perfusate. The HIPEC procedure takes $120 \mathrm{~min}$ in total, including the 90 -min perfusion period. To prevent heat trauma to normal tissue the temperature of the silicon drain will not be increased over $42^{\circ} \mathrm{C}$. Post-surgical phase: Urine production should not be less than $1 \mathrm{ml} / \mathrm{kg}$ during hyperthermic perfusion and for $3 \mathrm{~h}$ following surgery.

Patients will receive 4 additional post-operative cycles (8 weeks) of FLOT in the post-operative treatment phase. Post-operative treatment should start 6 to 8 weeks, but at maximum 12 weeks, after surgery.

In both arms, tumor assessments (CT or MRI) are performed before randomization prior to surgery, and then every 3 months (radiological tumor assessment) thereafter until progression/relapse, death, or end of followup. A change from CT into MRI in the follow up period is possible at any time. Also during tumor assessment visits and additionally after surgery blood (EDTAplasma, serum and whole blood for genomic DNA isolation (ccfDNA)) is collected for later translational research projects.

During treatment, clinical visits (blood cell counts, detection of toxicity) occur prior to every treatment dose. Safety of FLOT/ HIPEC will be monitored continuously by careful monitoring of all adverse events (AEs) and serious adverse events (SAEs) reported.

The phase III design starts with a safety run-in phase. Eight weeks after 20 patients had curatively intended resection in Arm B, an interim safety analysis is performed that shows feasibility, safety, and tolerability of Arm B planned. It is not planned to discontinue recruitment for the interim safety analysis (see Fig. 1).

\section{Measures of outcomes and assessments Primary outcome}

The primary efficacy objective of the study is to compare progression/disease-free survival (PFS/DFS), defined as first occurrence of progression or recurrence, as determined by the investigator using RECIST 1.1 criteria. The duration of PFS/DFS will be determined by measuring time interval from randomization until disease progression or disease recurrence after surgery or death of any cause.

\section{Secondary outcomes}

Secondary efficacy objectives are overall survival (OS, defined as the time from randomization to death from any cause), rates of peritoneal relapse at 2 and 3 years in both arms, PFS/DFS rates at 2, 3 and 5 years, OS rates at 3 and 5 years, OS and PFS/DFS (medians and rates) according to subgroups, Quality of life (QoL) - EORTC QLQ C30 and EORTC QLQ STO22 questionnaires, post-operative morbidity/mortality at day 30 after surgery acc. Clavien-Dindo classification, post-operative pain according to Visual analog scale and the safety of perioperative FLOT + HIPEC.

\section{Main inclusion criteria}

Histologically confirmed, medically operable, resectable diffuse or mixed type adenocarcinoma of the gastroesophageal junction (AEG II-III) or the stomach (uT3, uT4a, any $\mathrm{N}$ category, M0), or any $\mathrm{T} \mathrm{N}+\mathrm{M} 0$ patient is 


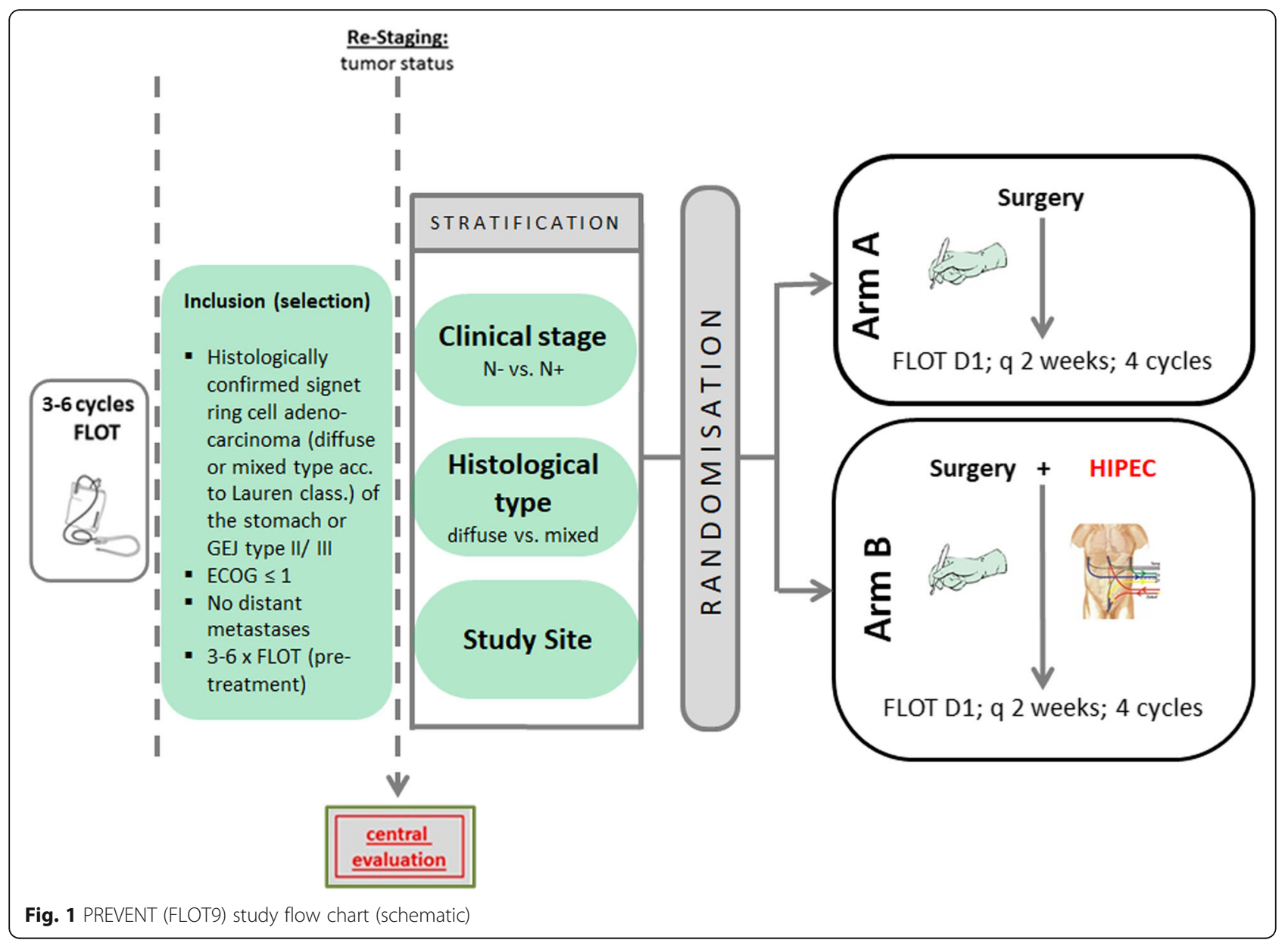

eligible. No prior chemotherapy except 3-6 cycles of FLOT chemotherapy and no prior tumor resection.

\section{Main exclusion criteria}

Medical inoperability. Inability to understand the study and/or comply with the protocol procedures. Preexisting peritoneal seeding.

Criteria of primary unresectability, e.g.: radiologically documented evidence of major blood vessel invasion or invasion of adjacent organs (T4b). Patient with involved retroperitoneal (e.g. para-aortal, paracaval or interaortocaval lymph nodes) or mesenterial lymph nodes (distant metastases).

\section{Treatments}

\section{Control(s)/comparator(s)}

FLOT consists of: Docetaxel $50 \mathrm{mg} / \mathrm{m}^{2}$, iv over $2 \mathrm{~h}$, d1; Oxaliplatin $85 \mathrm{mg} / \mathrm{m}^{2}$ in $500 \mathrm{ml} \mathrm{G} 5 \%$, iv over $2 \mathrm{~h}$, d1; Leucovorin $200 \mathrm{mg} / \mathrm{m}^{2}$ in $250 \mathrm{ml} \mathrm{NaCl} 0.9 \%$, iv over $1 \mathrm{~h}$, d1; 5 -FU $2600 \mathrm{mg} / \mathrm{m}^{2}$, iv over $24 \mathrm{~h}$, d1 (= 1 cycle); Start of next cycle on day 15 (every 2 weeks) [7].
Dose, mode, and scheme of intervention

In both arms, patients will undergo surgery 4 to 6 weeks after the 3-6th cycle of FLOT.

Surgery is carried out in kind of gastrectomy, transhiatal extended gastrectomy. In the experimental arm surgery will be combined with an intraoperative Hyperthermic IntraPEritoneal Chemoperfusion (HIPEC).

HIPEC itself can be performed in closed or openabdomen procedure. At start of hyperthermic perfusion, Sodium thiosulfate: $9 \mathrm{~g} / \mathrm{m}^{2}$ in $200 \mathrm{ml}$ distilled water, made isotone with sodium chloride, is to be given IV push over 15-20 min, concurrently at start of hyperthermic infusion of cisplatin. This is to be followed by $12 \mathrm{~g} /$ $\mathrm{m}^{2}$ thiosulfate IV continuous infusion over $6 \mathrm{~h}$ (the $12 \mathrm{~g} /$ $\mathrm{m}^{2}$ should be dissolved in 11 of distilled water and infused at $167 \mathrm{ml} / \mathrm{hr}$ ). After positioning of inflow catheter and drains intraabdominal cisplatin solution $\left(75 \mathrm{mg} / \mathrm{m}^{2}\right.$ in $\mathrm{NaCl} 0.9 \%$ ) will be administered at a temperature of $42{ }^{\circ} \mathrm{C}$ for $90 \mathrm{~min}$. Perfusion with cisplatin at a dose of 75 mg per square meter and at a flow rate of 11 per minute will be then initiated (with $50 \%$ of the dose perfused initially, $25 \%$ at $30 \mathrm{~min}$, and $25 \%$ at $60 \mathrm{~min}$ ). The perfusion volume will be adjusted such that the entire abdomen is 
exposed to the perfusate. The HIPEC procedure takes $120 \mathrm{~min}$ in total, including the 90 -min perfusion period. For a more exact description see above under: Arm B (FLOT/ HIPEC- experimental arm).

\section{Sample size calculation}

The primary efficacy analysis will compare randomized surgical resection combined with HIPEC to randomized surgical resection only on the time to the primary efficacy endpoint using the intent-to-treat population. The hypothesis test will use the log rank test to compare the investigational arms. The study assumes a Hazard ratio of 0.65 favoring the HIPEC group. The PFS/DFS in the reference arm is set as 20.19 months (calculation on complete data from study FLOT4) for signet ring cell containing gastric cancers. Accrual time is 42 months followed by 2 years follow up period. Dropouts prior to randomization are set at 35\%. Dropouts after randomization are set $5 \%$. Type I error is $5 \%$ and onesided Log rank test is used. Two hundred patients are to be randomized to provide a statistical power of $80 \%$. These 200 patients will be recruited in 20 German sites with already proven experience in conducting HIPEC procedure.

\section{Monitoring}

All adverse events and severe adverse events occurring after informed consent are recorded in the patient's electronic case report form by the responsible site staff. Adverse events will be assessed according to the Common Terminology Criteria for Adverse Events (CTCAE) version 5.0. With this data the safety will be monitored continuously by careful monitoring of all adverse events and serious adverse events reported. A compilation of all serious adverse events is sent to lead Ethic, regulatory body and the Safety Monitoring Board (SMB). The SMB furthermore provides the sponsor with recommendations regarding study modification, continuation or termination. In this process the SMB may give advice for continuation, changes to the study protocol or termination of the study. The SMB may claim unplanned interim analyses of any variable and - beyond the aforementioned items - it may ask for any additional activity within the trial if the activity is on behalf of patients' security.

Premature termination of the study may also be decided if unexpected severe surgical complications occur, more effective therapies become available or if patient enrollment is insufficient. Final decision is made by sponsor representative and the lead coordinating investigator.

It is understood that an outside monitor and other authorized personnel may contact and visit the investigator, and that they will be allowed direct access to source data/documents for trial-related monitoring, audits, IRB review, and regulatory inspection. Direct access is defined as permission to examine, analyze, verify, and reproduce any records and reports that are important to evaluation of a clinical trial. All reasonable precautions within the constraints of the applicable regulatory requirements to maintain the confidentiality of patients' identities and sponsor's proprietary information will be exercised. In case of an audit by the sponsor/sponsor representative or an appropriate authority, the investigator will make all relevant documents available.

\section{Ethical considerations, information giving and written informed consent}

The study protocol was approved by the responsible lead ethics committee on the July 27th, 2020 under the identification number 2020-1709-fAM. The study has been registered on the ClinicalTrial.gov website under the identification number NCT04447352 and under EudraCT 2017-003832-35. The PREVENT (FLOT9) study complies with the Declaration of Helsinki rules, the principles of Good Clinical Practice guidelines and the Data Protection Act. The trial will also be carried out in compliance to local legal and regulatory requirements. For each patient to be enrolled into the study, obtaining written informed consent prior to inclusion into the study is essential.

\section{Discussion}

Several Asian authors have reported a potential benefit from using intraperitoneal chemotherapy with or without hyperthermia, as an adjuvant therapy following curative surgery [19, 20]. Fujimoto et al. [21] recruited 141 patients and showed that HIPEC significantly reduced the incidence of peritoneal recurrence $(p<0.001)$ and increased the survival rate $(p=0.03)$ without a significant increase in postoperative adverse events. Yonemura et al. [22] showed in a randomized trial of 139 patients favorable 5-year survival rate in gastric cancer patients treated with HIPEC- therapy.

In 2001, Kim and Bae [23] published the results of a controlled study on 103 patients presenting with serosapositive gastric carcinoma, who underwent surgical resection alone or surgical resection combined with HIPEC with significantly higher 5-year survival rates in the HIPEC group, when stage IV patients were excluded. A meta-analysis by Yan et al. [24] demonstrated that using HIPEC as an adjuvant treatment significantly improved survival rates in stomach cancer and suggested that intraperitoneal chemotherapy + hyperthermia delivered during surgery was a more effective than a delayed approach. The meta-analysis of Coccolini confirmed the potential benefit of using HIPEC for patients with an advanced gastric cancer in an adjuvant setting [25]. 
However, all the studies mentioned above included mostly patients of Asian origin, where tumor biology, therapeutic strategies, and prognosis differ from those in Western countries. Moreover, the studies had significant shortcomings. The trials were underpowered, endpoints and statistics did not meet the modern quality criteria and the adjuvant chemotherapy regimens are not corresponding to the modern therapies of the western world using FLOT in the perioperative curative or even oligometastatic situation $[7,8,26]$.

The potential role of a so called prophylactic HIPEC and even laparoscopic neoadjuvant HIPEC are currently being increasingly used and evaluated. The combination of classic systemic chemotherapy with an intraperitoneal therapy gained popularity already in the last century in the late $90 \mathrm{~s}$, because of promising early results in several Phase II trials. Unfortunately, these findings could not be confirmed in e.g. recent trials with intraperitoneal approaches like the PHOENIX-gastric cancer study [27], a randomized controlled trial. The appropriate treatment in gastric cancer with high risk for peritoneal seeding or an already established low burden peritoneal carcinomatosis still remains controversial and a trial in this field of a high unmet need is required.

Peritoneal seeding in advanced gastric cancer patients is detected in up to $30 \%$ and associated with a poor prognosis $[28,29]$ measured by a median overall survival of 3-6 months without treatment and 6-12 months with chemotherapy [30-34].

Classical systemic chemotherapy has only a very limited effect caused by the problem of peritoneal plasma barrier with a reduced permeability of intravenous applicated agents with significantly reduced peritoneal concentration relative to plasma clearance [35], delivering a strong rationale for HIPEC- therapy due to direct delivery of appropriate doses of chemotherapy into the peritoneal cavity [35].

Hyperthermic chemotherapy is able to achieve deeper penetration in the peritoneal cavity and is able to enhance the antitumoral effects of chemotherapy directly at the region of interest [36].

The aim of HIPEC mostly in combination with cytoreductive surgery, is normally to remove all visible peritoneal seedings, while the heated therapy would treat residual microscopic disease.

In our current study population, there are no visible signs of a peritoneal seeding, based on the inclusion criteria and therefore there is no need for cytoreductive surgery but the need for heated chemotherapy to treat potential residual microscopic occult or incidental disease not visibly or cytologically detectable in a high risk group for peritoneal seeding, with the potential to cure. This will be tested in a population of 200 patients. This sample size takes into account the recent FLOT4 data.
A recent meta-analysis by Desiderio et al. [37] of 11 randomized controlled and 21 comparative non- randomized trials was able to show a significant, modest, amelioration in median overall survival with the addition of HIPEC to cytoreductive surgery (CRS) in gastric cancer (HIPEC+ CRS vs. CRS, median OS 11.1 vs. 7.1 months, $P<0.001$ ). Furthermore lots of individual studies reported that a low volume peritoneal seeding combined with complete cytoreduction are most likely to benefit from HIPEC- therapy, in selected patients [38-40].

In the meta-analysis of Desiderio et al. [37] the addition of HIPEC in patients with locally advanced cT3-4 disease and no evidence of a peritoneal spread demonstrated a decrease in overall disease recurrence (relative risk [RR], 0.73; 95\% CI, 0.59-0.89; $P=0.002$ ), 3year (RR, $0.71 ; 95 \% \mathrm{CI}, 0.53-0.96 ; P=0.03$ ) and 5 -year (RR, 0.82; 95\% CI, 0.70-0.96; $P=0.01$ ). A meta-analysis by Sun et al. [41] based on 10 randomized trials evaluated the addition of HIPEC to surgery for T4a gastric cancers, with no gross evidence of peritoneal metastatic implants, and reported a significant risk reduction in mortality (RR, $0.73 ; 95 \% \mathrm{CI}, 0.64-0.83 ; P<0.001$ ) as well peritoneal recurrence (RR, $0.45 ; 95 \% \mathrm{CI}, 0.28-0.72$; $P=0.001$ ).

The mentioned data provide a strong rationale for a prophylactic HIPEC- approach. The ongoing GASTRICHIP- trial (NCT01882933) [42] is currently recruiting patients with T3/4 gastric tumors, irrespective of nodal or peritoneal cytology status, and randomizing patients to undergo either gastrectomy alone or with HIPEC.

The approach of PREVENT (FLOT9) is even more specific, only recruiting patients with high risk for developing peritoneal metastatic disease, respecting the nature of mixed and diffuse type gastric cancer seeding into the peritoneal cavity compared with the intestinal type where hematogenous spread with a hepatic seeding is more common. Due to respecting the molecular nature of the different gastric cancer types, intestinal type gastric cancers are excluded in our approach, because in future tailored trials it seems to be important not to treat the entire gastric population, but the right pts. to have the possible opportunity for a positive trial with the correct method.

Patients with $\mathrm{GC}>\mathrm{T} 2$, diffuse or mixed type histological subtype, and lymphovascular invasion are at increased risk of peritoneal relapse, but at this stage still with a potential for cure. However, the benefit of prophylactic HIPEC after radical gastrectomy in these patients remains controversial [43] and needs further evaluation within a randomized trial like the current PREVENT (FLOT9).

\section{Abbreviations}

ACO: Assoziation chirurgische Onkologie; AIO: Arbeitsgemeinschaft Internistische Onkologie- Working group of Medical Oncologists; 
CAOGI: Chirurgische Arbeitsgemeinschaft für den Oberen Gastrointestinaltrakt; EORTC: European Organization for Research and Treatment of Cancer; EORTC-QLQ-C30: European Organization for Research and Treatment of Cancer - Quality of Life Questionnaire-C30; EORTC-QLQ STO22: European Organization for Research and Treatment of Cancer Quality of Life Questionnaire-Stomach 22 questions; FLOT: 5-flourouracil, leucovorin, oxaliplatin, and docetaxel; GEJ: Gastroesophageal junction cancer; QoL: Quality of life; HIPEC: Hyperthermic IntraPEritoneal Chemoperfusion

\section{Acknowledgements}

The authors thank Dr. rer. nat. Axel Hinke for statistical advice and the Deutsche Krebshilfe (German Cancer Aid) as the funding source and the AlO Arbeitsgemeinschaft Internistische Onkologie (Working group of Medical Oncologists), the Chirurgische Arbeitsgemeinschaft für den Oberen Gastrointestinaltrakt (CAOGI) and the Assoziation chirurgische Onkologie (ACO) for study support.

\section{Authors' contributions}

$\mathrm{TG}, \mathrm{AB}$ and $\mathrm{UB}$ have been involved in the study conception and design and in drafting the manuscript. All authors (TG, PP, SL, UB, CP, ME, GA, DR, WB, $A K, S M, B R, M S, A B$ ) read and approved the final manuscript. All 14 authors of the manuscript made substantial contributions in acquisition of data and have been involved in revising the manuscript critically for important intellectual content. Each of the 14 authors has given final approval of the version to be published and have participated sufficiently in the work to take public responsibility for appropriate portions of the content and agreed to be accountable for all aspects of the work in ensuring that questions related to the accuracy or integrity of any part of the work are appropriately investigated and resolved.

\section{Authors' information}

TG is the study coordinator, obtained the grant and is responsible for the present paper.

\section{Funding}

The PREVENT study is funded by the Deutsche Krebshilfe (German Cancer Aid). The identification number of the funding source is 70113137 . The funding body has peer reviewed the trial protocol and has no influence in the collection, analysis, interpretation of data or the design of the study. Also the writing of manuscript was not influenced by the funding body.

\section{Availability of data and materials}

"Not applicable".

\section{Declarations}

\section{Ethics approval and consent to participate}

The study protocol was approved by the responsible lead ethics committee of the Landesärtzekammer Hessen, Hanauer Landstr. 152, 60314 Frankfurt am Main, Germany, responsible for the Krankenhaus Nordwest Frankfurt- UCT (University Cancer Center) on July 27th, 2020 under the identification number 2020-1709-fAM. A trial amendment was approved by the responsible lead ethics committee on May 18th, 2021, Version 1.2. The study has been registered on the ClinicalTrial.gov website under the identification number NCT04447352. The PREVENT study complies with the Declaration of Helsinki rules, the principles of Good Clinical Practice guidelines and the Data Protection Act. The trial will also be carried out in compliance to local legal and regulatory requirements. For each patient to be enrolled into the study, obtaining written informed consent prior to inclusion into the study is essential.

\section{Consent for publication}

"Not applicable".

\section{Competing interests}

The authors declare that they have no competing interests.

\section{Author details}

'Institute of Clinical Cancer Research (IKF) at Krankenhaus Nordwest, UCT-University Cancer Center, Frankfurt, Germany. ${ }^{2}$ Institut für Klinische Krebsforschung IKF GmbH am Krankenhaus Nordwest, Frankfurt, Germany.
${ }^{3}$ Department for General and Visceral Surgery, Hospital Barmherzige Brueder, University of Regensburg, Regensburg, Germany. ${ }^{4}$ Third Department of Internal Medicine (Hematology/Medical Oncology), Klinikum Rechts der Isar, Technische Universitat Munchen, Munich, Germany. ${ }^{5}$ Bariatrische und Metabolische Chirurgie, Krankenhaus Nordwest, Frankfurt, Germany. ${ }^{6}$ Department of General Surgery and Emergency, University of Palermo, Palermo, Italy. ${ }^{7}$ Klinik und Poliklinik für Chirurgie, Klinikum rechts der Isar, Technische Universität München, Munich, Germany. ${ }^{8}$ Department of General and Visceral Surgery, University Hospital Frankfurt, Frankfurt, Germany. ${ }^{9}$ Department of General-, Visceral Surgery and Transplantation, University Hospital Tübingen, Tübingen, Germany. ${ }^{10}$ Service de Chirurgie viscérale, Hôpitaux Universitaires de Genève, Geneva, Switzerland. " ${ }^{1}$ Department of Surgery, Campus Charité Mitte, Campus Virchow-Klinikum CCM/CVK, Berlin, Germany. ${ }^{12}$ Clinic for General, Visceral, Vascular and Thoracic Surgery, Klinikum Frankfurt Höchst, Frankfurt, Germany.

Received: 29 July 2021 Accepted: 11 October 2021

Published online: 29 October 2021

\section{References}

1. Duarte I, Llanos O. Patterns of metastases in intestinal and diffuse types of carcinoma of the stomach. Hum Pathol. 1981;12(3):237-42. https://doi.org/1 0.1016/S0046-8177(81)80124-4.

2. Esaki Y, Hirayama R, Hirokawa K. A comparison of patterns of metastasis in gastric cancer by histologic type and age. Cancer. 1990;65(9):2086-90. https://doi.org/10.1002/1097-0142(19900501)65:9<2086::AID-CNCR282 0650933>3.0.CO;2-B

3. Haraguchi M, Okamura T, Korenaga D, Tsujitani S, Marin P, Sugimachi K. Heterogeneity of DNA ploidy in patients with undifferentiated carcinomas of the stomach. Cancer. 1987;59(5):922-4. https://doi.org/10.1002/1097-0142 (19870301)59:5<922::AID-CNCR2820590511>3.0.CO;2-0.

4. Maehara Y, Moriguchi S, Orita H, Kakeji Y, Haraguchi M, Korenaga D, et al. Lower survival rate for patients with carcinoma of the stomach of Borrmann type IV after gastric resection. Surg Gynecol Obstet. 1992;175(1):13-6.

5. Rhomberg W, Gruber U. Liver metastasis in cancer of the stomach and its dependence on the histology of the primary tumor: an autopsy study on 102 cases. Clin Exp Metastasis. 1989;7(6):585-90. https://doi.org/10.1007/ BF01753669.

6. Al-Shammaa HA, Li Y, Yonemura Y. Current status and future strategies of cytoreductive surgery plus intraperitoneal hyperthermic chemotherapy for peritoneal carcinomatosis. World J Gastroenterol. 2008;14(8):1159-66. https://doi.org/10.3748/wjg.14.1159

7. Al-Batran SE, Hofheinz RD, Pauligk C, Kopp HG, Haag GM, Luley KB, et al. Histopathological regression after neoadjuvant docetaxel, oxaliplatin, fluorouracil, and leucovorin versus epirubicin, cisplatin, and fluorouracil or capecitabine in patients with resectable gastric or gastro-oesophageal junction adenocarcinoma (FLOT4-AIO): results from the phase 2 part of a multicentre, open-label, randomised phase 2/3 trial. Lancet Oncol. 2016; 17(12):1697-708. https://doi.org/10.1016/s1470-2045(16)30531-9.

8. Al-Batran SE, Homann N, Pauligk C, Goetze TO, Meiler J, Kasper S, et al. Perioperative chemotherapy with fluorouracil plus leucovorin, oxaliplatin, and docetaxel versus fluorouracil or capecitabine plus cisplatin and epirubicin for locally advanced, resectable gastric or gastro-oesophageal junction adenocarcinoma (FLOT4): a randomised, phase 2/3 trial. Lancet. 2019;393(10184):1948-57. https://doi.org/10.1016/s0140-6736(18)32557-1.

9. Al-Batran SE, Hartmann JT, Probst S, Schmalenberg H, Hollerbach S, Hofheinz R, et al. Phase III trial in metastatic gastroesophageal adenocarcinoma with fluorouracil, leucovorin plus either oxaliplatin or cisplatin: a study of the Arbeitsgemeinschaft Internistische Onkologie. J Clin Oncol. 2008;26(9):1435-42. https://doi.org/10.1200/JCO.2007.13.9378.

10. Al-Batran SE, Homann N, Pauligk C, Illerhaus G, Martens UM, Stoehlmacher J, et al. Effect of neoadjuvant chemotherapy followed by surgical resection on survival in patients with limited metastatic gastric or gastroesophageal junction cancer: the AIO-FLOT3 trial. JAMA Oncol. 2017b;3(9):1237-44. https://doi.org/10.1001/jamaoncol.2017.0515.

11. Al-Batran SE, Pauligk C, Homann N, Hartmann JT, Moehler M, Probst S, et al. The feasibility of triple-drug chemotherapy combination in older adult patients with oesophagogastric cancer: a randomised trial of the Arbeitsgemeinschaft Internistische Onkologie (FLOT65+). Eur J Cancer. 2013; 49(4):835-42. https://doi.org/10.1016/j.ejca.2012.09.025. 
12. van Driel WJ, Koole SN, Sikorska K, Schagen van Leeuwen JH, Schreuder HWR, Hermans RHM, et al. Hyperthermic intraperitoneal chemotherapy in ovarian cancer. N Engl J Med. 2018;378(3):230-40. https://doi.org/10.1056/ NEJMoa1708618.

13. Tanis PJ, Tuynman JB, de Hingh I. Results from the PROPHYLOCHIPPRODIGE 15 trial. Lancet Oncol. 2020;21(11):e496. https://doi.org/10.1016/s14 70-2045(20)30497-6

14. Quénet F, Elias D, Roca L, Goéré D, Ghouti L, Pocard M, et al. Cytoreductive surgery plus hyperthermic intraperitoneal chemotherapy versus cytoreductive surgery alone for colorectal peritoneal metastases (PRODIGE 7): a multicentre, randomised, open-label, phase 3 trial. Lancet Oncol. 2021; 22(2):256-66. https://doi.org/10.1016/s1470-2045(20)30599-4.

15. Liu Y, Yonemura Y, Levine EA, Glehen O, Goere D, Elias D, et al. Cytoreductive surgery plus hyperthermic intraperitoneal chemotherapy for peritoneal metastases from a small bowel adenocarcinoma: multiinstitutional experience. Ann Surg Oncol. 2018;25(5):1184-92. https://doi org/10.1245/s10434-018-6369-x.

16. Schmiegel W, Buchberger B, Follmann M, Graeven U, Heinemann V, Langer T, et al. Not available. Z Gastroenterol. 2017:55(12):1344-498. https://doi. org/10.1055/s-0043-121106

17. S3-Leitlinie Kolorektales Karzinom. Leitlinienprogramm Onkologie S3-Leitlinie Kolorektales Karzinom August 2014. 2014.

18. Glehen O, Gilly FN, Arvieux C, Cotte E, Boutitie F, Mansvelt B, et al. Peritoneal carcinomatosis from gastric cancer: a multi-institutional study of 159 patients treated by cytoreductive surgery combined with perioperative intraperitoneal chemotherapy. Ann Surg Oncol. 2010;17(9):2370-7. https:// doi.org/10.1245/s10434-010-1039-7.

19. Xu DZ, Zhan YQ, Sun XW, Cao SM, Geng QR. Meta-analysis of intraperitoneal chemotherapy for gastric cancer. World J Gastroenterol. 2004;10(18):2727-30. https://doi.org/10.3748/wjg.v10.i18.2727.

20. Yu W, Whang I, Suh I, Averbach A, Chang D, Sugarbaker PH. Prospective randomized trial of early postoperative intraperitoneal chemotherapy as an adjuvant to resectable gastric cancer. Ann Surg. 1998;228(3):347-54. https:// doi.org/10.1097/00000658-199809000-00007.

21. Fujimoto S, Takahashi M, Mutou T, Kobayashi K, Toyosawa T. Successful intraperitoneal hyperthermic chemoperfusion for the prevention of postoperative peritoneal recurrence in patients with advanced gastric carcinoma. Cancer. 1999;85(3):529-34. https://doi.org/10.1002/(SICI)10970142(19990201)85:3<529::AID-CNCR3>3.0.CO;2-9.

22. Yonemura $Y$, de Aretxabala X, Fujimura T, Fushida S, Katayama K, Bandou E, et al. Intraoperative chemohyperthermic peritoneal perfusion as an adjuvant to gastric cancer: final results of a randomized controlled study. Hepatogastroenterology. 2001;48(42):1776-82.

23. Kim JY, Bae HS. A controlled clinical study of serosa-invasive gastric carcinoma patients who underwent surgery plus intraperitoneal hyperthermo-chemo-perfusion (IHCP). Gastric Cancer. 2001;4(1):27-33. https://doi.org/10.1007/s101200100013.

24. Yan TD, Black D, Sugarbaker PH, Zhu J, Yonemura Y, Petrou G, et al. A systematic review and meta-analysis of the randomized controlled trials on adjuvant intraperitoneal chemotherapy for resectable gastric cancer. Ann Surg Oncol. 2007;14(10):2702-13. https://doi.org/10.1245/s10434-007-9487-4.

25. Coccolini F, Cotte E, Glehen O, Lotti M, Poiasina E, Catena F, et al. Intraperitoneal chemotherapy in advanced gastric cancer. Meta-analysis of randomized trials. Eur J Surg Oncol. 2014;40(1):12-26. https://doi.org/10.101 6/j.ejso.2013.10.019.

26. Al-Batran SE, Goetze TO, Mueller DW, Vogel A, Winkler M, Lorenzen S, et al. The RENAISSANCE (AIO-FLOT5) trial: effect of chemotherapy alone vs. chemotherapy followed by surgical resection on survival and quality of life in patients with limited-metastatic adenocarcinoma of the stomach or esophagogastric junction - a phase III trial of the German AIO/CAO-V/ CAOGI. BMC Cancer. 2017a;17(1):893. https://doi.org/10.1186/s12885-017-391 $8-9$

27. Ishigami H, Fujiwara Y, Fukushima R, Nashimoto A, Yabusaki H, Imano M, et al. Phase III trial comparing intraperitoneal and intravenous paclitaxel plus S-1 versus cisplatin plus S-1 in patients with gastric cancer with peritoneal metastasis: PHOENIX-GC trial. J Clin Oncol. 2018;36(19):1922-9. https://doi. org/10.1200/jco.2018.77.8613.

28. Jacquet P, Sugarbaker PH. Peritoneal-plasma barrier. Cancer Treat Res. 1996; 82:53-63. https://doi.org/10.1007/978-1-4613-1247-5_4.

29. Yonemura Y, Canbay E, Li Y, Coccolini F, Glehen O, Sugarbaker PH, et al. A comprehensive treatment for peritoneal metastases from gastric cancer with curative intent. Eur J Surg Oncol. 2016;42(8):1123-31. https://doi.org/1 0.1016/j.ejso.2016.03.016

30. Chu DZ, Lang NP, Thompson C, Osteen PK, Westbrook KC. Peritoneal carcinomatosis in nongynecologic malignancy. A prospective study of prognostic factors. Cancer. 1989;63(2):364-7. https://doi.org/10.1002/10970142(19890115)63:2<364::aid-cncr2820630228>3.0.co;2-v.

31. Oh SY, Kwon HC, Lee S, Lee DM, Yoo HS, Kim SH, et al. A phase II study of oxaliplatin with low-dose leucovorin and bolus and continuous infusion 5fluorouracil (modified FOLFOX-4) for gastric cancer patients with malignant ascites. Jpn J Clin Oncol. 2007;37(12):930-5. https://doi.org/10.1093/jjco/ hym131.

32. Sadeghi B, Arvieux C, Glehen O, Beaujard AC, Rivoire M, Baulieux J, et al. Peritoneal carcinomatosis from non-gynecologic malignancies: results of the EVOCAPE 1 multicentric prospective study. Cancer. 2000;88(2):358-63. https://doi.org/10.1002/(sici)1097-0142(20000115)88:2<358:.aid-cncr16>3.0. $\mathrm{co} ; 2-\mathrm{O}$.

33. Shirao K, Boku N, Yamada Y, Yamaguchi K, Doi T, Goto M, et al. Randomized phase III study of 5-fluorouracil continuous infusion vs. sequential methotrexate and 5-fluorouracil therapy in far advanced gastric cancer with peritoneal metastasis (JCOG0106). Jpn J Clin Oncol. 2013;43(10):972-80. https://doi.org/10.1093/jjco/hyt114.

34. Yamao T, Shimada Y, Shirao K, Ohtsu A, Ikeda N, Hyodo I, et al. Phase II study of sequential methotrexate and 5-fluorouracil chemotherapy against peritoneally disseminated gastric cancer with malignant ascites: a report from the Gastrointestinal Oncology Study Group of the Japan Clinical Oncology Group, JCOG 9603 trial. Jpn J Clin Oncol. 2004;34(6):316-22. https://doi.org/10.1093/jjco/hyh063.

35. Dedrick RL, Myers CE, Bungay PM, DeVita VT Jr. Pharmacokinetic rationale for peritoneal drug administration in the treatment of ovarian cancer. Cancer Treat Rep. 1978;62(1):1-11.

36. Witkamp AJ, de Bree E, Van Goethem R, Zoetmulder FA. Rationale and techniques of intra-operative hyperthermic intraperitoneal chemotherapy. Cancer Treat Rev. 2001:27(6):365-74. https://doi.org/10.1053/ctrv.2001.0232.

37. Desiderio J, Chao J, Melstrom L, Warner S, Tozzi F, Fong Y, et al. The 30-year experience-a meta-analysis of randomised and high-quality non-randomised studies of hyperthermic intraperitoneal chemotherapy in the treatment of gastric cancer. Eur J Cancer. 2017;79:1-14. https://doi.org/10.1016/j.ejca.2017. 03.030 .

38. Hall JJ, Loggie BW, Shen P, Beamer S, Douglas Case L, McQuellon R, et al. Cytoreductive surgery with intraperitoneal hyperthermic chemotherapy for advanced gastric cancer. J Gastrointest Surg. 2004;8(4):454-63. https://doi. org/10.1016/j.gassur.2003.12.014

39. Yang XJ, Huang CQ, Suo T, Mei LJ, Yang GL, Cheng FL, et al. Cytoreductive surgery and hyperthermic intraperitoneal chemotherapy improves survival of patients with peritoneal carcinomatosis from gastric cancer: final results of a phase III randomized clinical trial. Ann Surg Oncol. 2011;18(6):1575-81. https://doi.org/10.1245/s10434-011-1631-5.

40. Yarema RR, Ohorchak MA, Zubarev GP, Mylyan YP, Oliynyk YY, Zubarev MG, et al. Hyperthermic intraperitoneal chemoperfusion in combined treatment of locally advanced and disseminated gastric cancer: results of a singlecentre retrospective study. Int J Hyperth. 2014;30(3):159-65. https://doi.org/1 0.3109/02656736.2014.893451.

41. Sun J, Song Y, Wang Z, Gao P, Chen X, Xu Y, et al. Benefits of hyperthermic intraperitoneal chemotherapy for patients with serosal invasion in gastric cancer: a meta-analysis of the randomized controlled trials. BMC Cancer. 2012;12(1):526. https://doi.org/10.1186/1471-2407-12-526.

42. Glehen O, Passot G, Villeneuve L, Vaudoyer D, Bin-Dorel S, Boschetti G, et al. GASTRICHIP: D2 resection and hyperthermic intraperitoneal chemotherapy in locally advanced gastric carcinoma: a randomized and multicenter phase III study. BMC Cancer. 2014;14(1):183. https://doi.org/10.1186/1471-2407-14-1 83.

43. Pasqual EM, Bertozzi S, Londero AP, Brandolin D, Mariuzzi L, De Pellegrin A, et al. Microscopic peritoneal carcinomatosis in gastric cancer: prevalence, prognosis and predictive factors. Oncol Lett. 2018;15(1):710-6. https://doi. org/10.3892/ol.2017.7442.

\section{Publisher's Note}

Springer Nature remains neutral with regard to jurisdictional claims in published maps and institutional affiliations. 